\title{
Transfer of triptans into human breast milk and estimation of infant drug exposure through breastfeeding
}

\author{
Siri Amundsen $^{1,2}$ (D) | Hedvig Nordeng ${ }^{3}$ (D) | Ole-Martin Fuskevåg ${ }^{1,2}$ (i) | \\ Elisabet Nordmo $^{4}$ | Georg Sager ${ }^{2}$ (i) | Olav Spigset ${ }^{5,6}$
}

\author{
${ }^{1}$ Department of Laboratory Medicine, \\ University Hospital of North Norway, \\ Troms $\varnothing$, Norway \\ ${ }^{2}$ Experimental and Clinical Pharmacology \\ Research Group, Department of Medical \\ Biology, Faculty of Health Sciences, UiT - \\ The Arctic University of Norway, Troms $\varnothing$, \\ Norway \\ ${ }^{3}$ Pharmacoepidemiology and Drug Safety \\ Research Group, Department of Pharmacy, \\ University of Oslo, Oslo, Norway \\ ${ }^{4}$ Regional Medicines Information and \\ Pharmacovigilance Centre (RELIS), \\ University Hospital of North Norway, \\ Tromsø, Norway \\ ${ }^{5}$ Department of Clinical Pharmacology, \\ St. Olav University Hospital, Trondheim, \\ Norway \\ ${ }^{6}$ Department of Clinical and Molecular \\ Medicine, Norwegian University of Science \\ and Technology, Trondheim, Norway

\section{Correspondence} \\ Siri Amundsen, Department of Laboratory \\ Medicine, University Hospital of North \\ Norway, PO box 63, N-9038 Troms $\varnothing$, Norway. \\ Email: siri.amundsen@unn.no \\ Funding information \\ Helse Nord RHF, Grant/Award Number: \\ Project ID: SFP1248-15
}

\begin{abstract}
Clinical data on the transfer of triptans into human breast milk remain scarce. In a lactation study including 19 breastfeeding women with migraine, we examined the excretion of six different triptans into milk. Following intake of a single dose, each participant collected seven breast milk samples at predefined intervals up to 24 hours after dose. Triptan concentrations in milk were measured using liquid chromatography-tandem mass spectrometry (LC-MS/MS). Infant drug exposure was estimated by calculating the relative infant dose (RID). Twenty-two breast milk sample sets were obtained for sumatriptan $(n=8)$, rizatriptan $(n=5)$, zolmitriptan $(n=4)$, eletriptan $(n=3)$, almotriptan $(n=1)$ and naratriptan $(n=1)$. Based on the average concentration in milk throughout the day, estimated mean RIDs (with range in parenthesis) were as follows: eletriptan $0.6 \%$ (0.3\%-0.8\%), sumatriptan $0.7 \%$ (0.2\%-1.8\%), rizatriptan $0.9 \%(0.3 \%-1.4 \%)$, almotriptan $1.8 \%(-)$, zolmitriptan $2.1 \%$ $(0.7 \%-5.3 \%)$ and naratriptan $5.0 \%$ (-). Infant drug exposure through breastfeeding appears to be low and indicates that use of the triptans in this study is compatible with breastfeeding. Naratriptan may not be first choice in breastfeeding mothers initiating triptans during the neonatal period.
\end{abstract}

\section{K E Y W O R D S}

breast milk, breastfeeding, lactation study, migraine, pharmacokinetics, relative infant dose, triptans

\section{1 | INTRODUCTION}

Triptans are selective serotonin $5-\mathrm{HT}_{1 \mathrm{~B} / 1 \mathrm{D}}$ receptor agonists commonly used for the acute treatment of migraine headaches and are often considered as first-line therapy for patients with moderate to severe migraine. ${ }^{1}$ Seven triptans are currently available worldwide: almotriptan, eletriptan, frovatriptan, naratriptan, rizatriptan, sumatriptan and zolmitriptan. These are rapid- and short-acting drugs due to their pharmacokinetic properties. ${ }^{2}$

Clinical data on the use of triptans in breastfeeding women are extremely limited, and their safety profile during breastfeeding is not established. Although many women with migraine 
experience an improvement of their disease during pregnancy, the recurrence of symptoms generally appears shortly following delivery. ${ }^{3-5}$ Making recommendations about the use of triptans during breastfeeding is at present hindered by the limited data available, possibly leaving many women with suboptimal migraine treatment during this period. Since the marketing of sumatriptan, as the first drug of this class, in the early 1990s, only two studies have examined the excretion of triptans into human breast milk, one for sumatriptan ${ }^{6}$ and one for eletriptan. ${ }^{7}$

Wojnar-Horton et $a l^{6}$ measured the concentration of sumatriptan in breast milk and plasma of five lactating women given a 6-mg-dose by subcutaneous injection. The mean milk/plasma $(\mathrm{M} / \mathrm{P})$ ratio was 4.9 . The mean total recovery of sumatriptan excreted in milk over the 8-hour collection period was $14.4 \mu \mathrm{g}$, which equals $0.24 \%$ of the administered maternal dose. This corresponds to a mean relative infant dose (RID) of $3.5 \%$ of the maternal weight-adjusted dose. Considering that the drug is not given continuously and that sumatriptan has low oral availability (14\%), the authors concluded that sumatriptan exposure through breastfeeding is not likely to cause any pharmacological effects in the breastfed infant. ${ }^{6}$

In an unpublished study conducted by the manufacturer and briefly cited in a handbook, ${ }^{7}$ the excretion of eletriptan in breast milk was investigated in eight women following intake of a single oral dose of $80 \mathrm{mg}$. The $\mathrm{M} / \mathrm{P}$ ratio was 0.25 , and the mean total dose excreted in breast milk over the 24-hour collection period was $12.9 \mu \mathrm{g}$, or $0.02 \%$ of the administered maternal dose. Low concentrations (mean $1.7 \mathrm{ng} / \mathrm{mL}$ ) were still present in the milk 18-24 hours after dose. The active metabolite N-desmethyleletriptan was not analysed in the breast milk, neither were infant dose estimates reported in this study. ${ }^{7}$

No adverse effects in infants exposed to triptans through breastfeeding have been reported in the scientific literature. Despite little theoretical grounds for concern, the currently available documentation is not sufficient for giving conclusive advice. Thus, the product monographs for these medications generally include a warning about use during breastfeeding (see Table S1). At this time, sumatriptan is recommended as the preferred triptan for use in breastfeeding women. ${ }^{7}$

The aim of the present study was to quantify the excretion of almotriptan, eletriptan, naratriptan, rizatriptan, sumatriptan and zolmitriptan and relevant active metabolites (ie $\mathrm{N}$ desmethyleletriptan and $\mathrm{N}$-desmethylzolmitriptan), into breast milk. In addition, we estimated potential infant drug exposure through breastfeeding.

\section{MATERIALS AND METHODS}

\section{1 | Study participants}

Breastfeeding women with migraine reporting use of any of the six triptans under study were eligible to participate. We excluded women using frovatriptan due to ethical considerations related to its prolonged elimination half-life (26 hours), causing a possibly higher potential for adverse drug reactions in breastfed infants. Inclusion criteria were age $\geq 18$ years, being at least one month postpartum, and having established breastfeeding. The study period lasted from December 2015 to December 2017. Women were recruited through advertising on the website of the Norwegian teratology information service for the public; SafeMotherMedicine (www.tryggmamma medisin.no), a free-of-charge online service providing answers about medication use during pregnancy and breastfeeding. Information about the study was also posted on selected Norwegian websites specifically directed towards pregnant women and new mothers. In addition, posters with information of the study were distributed to paediatric clinics and midwife centres in the city of Troms $\varnothing$ in Northern Norway.

\subsection{Ethics and approvals}

The study was approved by the Regional Committee for Medical and Health Research Ethics in Northern Norway (2013/1757) and the Data Protection Officer at the University Hospital of North Norway (UNN). All women gave their written informed consent prior to inclusion in the study. The study was conducted in accordance with the Basic \& Clinical Pharmacology \& Toxicology policy for experimental and clinical studies ${ }^{8}$ and designed in line with the US Food and Drug Administration (FDA) recommendations for clinical lactation studies. ${ }^{9}$

\section{3 | Milk sampling procedure}

The participants were supplied with a kit including all necessary sampling equipment (sample containers, label stickers and a breast pump, if needed), and they conducted the sampling at home. When using the triptan prescribed by their doctor, they expressed mid-feed breast milk samples (approximately $5 \mathrm{~mL}$ from each breast) at the following predefined time intervals: 0 hour (pre-dose), 1, 2, 4, 8, 12 and 24 hours after dose intake, respectively. The samples were collected by use of a manual or electric breast pump, as preferred by the mother. The women were instructed to seal each container tightly and to freeze the milk samples in their home freezer immediately after sampling. We recommended the women to bottle-feed their infants with previously expressed breast milk for a period after dose equal to five elimination half-lives of the triptan administered to ensure no drug exposure to the infant.

The women recorded the date and time of dose intake, the date and time of each milk sampling, and labelled each of the containers correspondingly. In addition, they filled out a questionnaire covering maternal age, body-weight, height, a 
complete list of any other medication(s) used, and the infant's age, body-weight and mode of nutrition (fully breastfed, partially breastfed, formula-fed, eating solid food etc).

After the last breast milk sample was collected, the frozen samples were transported in insulated containers with frozen gel packs and delivered by express courier service to the Department of Laboratory Medicine, UNN, Tromsø. Upon arrival, the samples were examined to make sure that they were still in a frozen state, and they were subsequently stored at $-35^{\circ} \mathrm{C}$ until the day of analysis. All sample sets were analysed within a week after being received. Prior to the study, we performed a stability analysis of human breast milk samples spiked with the six triptans to evaluate whether temperature impacted drug stability in milk. Triplicates of each triptan were stored at ambient temperature $\left(22^{\circ} \mathrm{C}\right)$ for 24 hours, and $4^{\circ} \mathrm{C}$ for 24 hours and 8 days, before reanalysis of the samples. The stability proved satisfactory for all drugs, with no or only minor reductions in concentration (up to 5\% for the samples stored at $4^{\circ} \mathrm{C}$ for 8 days), compared with baseline (0 hours).

\subsection{Quantification of triptan concentrations in breast milk}

Quantification of the triptan and metabolite concentrations in milk was performed with liquid chromatography-tandem mass spectrometry (LC-MS/MS). The analytical method was developed and validated at the Department of Laboratory Medicine, UNN, Troms $\varnothing$.

\subsubsection{Chemicals and solutions}

Sumatriptan and zolmitriptan were purchased from LGC $\mathrm{GmbH}$, Luckenwalde, Germany. Almotriptan, eletriptan, naratriptan, rizatriptan and the deuterated almotriptan-d6, naratriptan-d3, rizatriptan-d6, sumatriptan-d6 and zolmitriptan-d6 were purchased from Toronto Research Chemicals Inc. N-desmethyleletriptan and $\mathrm{N}$-desmethylzolmitriptan were purchased from Santa Cruz Biotechnology. Blank human donor milk for the set-up of the analytical method and for the quality control (QC) samples was supplied by the Mothers' Milk Bank, UNN, Tromsø. LC-MS grade methanol and formic acid were purchased from Fluka (Sigma-Aldrich). Ultrapure water (18.2 M $\Omega$ ) was obtained from a Millipore Advantage Milli-Q system (Millipore SAS).

\subsection{2 | Liquid-liquid extraction}

Milk samples, spiked standards and QC samples were extracted as follows: $50 \mu \mathrm{L}$ sample, $25 \mu \mathrm{L}$ internal standard solution, $25 \mu \mathrm{L} 0.1 \mathrm{~mol} / \mathrm{L} \mathrm{Na}_{2} \mathrm{CO}_{3}(\mathrm{pH} \mathrm{11})$ and $1 \mathrm{~mL}$ n-chlorobutane:acetonitrile (4:1) were mixed and centrifuged. The organic phase was pipetted off, transferred to new tubes and evaporated to dryness under a stream of nitrogen at $40^{\circ} \mathrm{C}$, and the remaining extracts were reconstituted in $100 \mu \mathrm{L}$ water:methanol (95:5) with $0.1 \%$ formic acid.

\subsubsection{Liquid chromatography-tandem mass spectrometry}

Samples were analysed by LC-MS/MS using a Waters Acquity UPLC system with an autosampler and a binary solvent delivery system (Waters) interfaced to Waters Xevo TQ-S benchtop tandem quadrupole mass spectrometer (Waters). Separation was performed on a $2.1 \times 100 \mathrm{~mm}$ Waters Acquity HSS T3 column maintained at $50^{\circ} \mathrm{C}$.

Lower limit of quantification (LLOQ) was found to be $0.25 \mathrm{nM}$ for almotriptan and naratriptan, $0.5 \mathrm{nM}$ for sumatriptan, zolmitriptan and rizatriptan, and $1 \mathrm{nM}$ for eletriptan, N-desmethyleletriptan and $\mathrm{N}$-desmethylzolmitriptan. For a more detailed description of the LC-MS/MS method, preparation of calibrators and controls, and precision and accuracy, see Appendix S1.

\section{5 | Pharmacokinetic analysis}

Maximum milk drug concentration $\left(C_{\max }\right)$ and the corresponding time to maximum concentration $\left(t_{\max }\right)$ were obtained directly from the measured values. Other pharmacokinetic variables were calculated using the pharmacokinetic program package Kinetica, version 5.1 (Thermo Fisher Scientific). Area under the milk concentration-time curve (AUC) was calculated using a mixed log-linear model with extrapolation to infinity. The parameter estimate describing the decrease of the log-concentration $\left(\lambda_{\mathrm{z}}\right)$ was calculated using the best-fit log-linear regression line of the samples representing the elimination phase. The elimination half-life $\left(t_{1 / 2}\right)$ was calculated as $\ln 2 / \lambda_{\mathrm{z}}$. Data were presented graphically using SigmaPlot, version 14.0 (Systat Software).

\subsection{Estimation of infant drug exposure}

We estimated the theoretical weight-adjusted absolute infant dose (AID) $(\mu \mathrm{g} / \mathrm{kg} /$ day) received through breast milk if breastfeeding had continued post-dose, by multiplying the milk drug concentration $(\mu \mathrm{g} / \mathrm{L})$ by the daily milk intake, assuming an average daily infant milk intake of $150 \mathrm{~mL} / \mathrm{kg} .{ }^{10}$ For these calculations, we used the average triptan concentration $\left(C_{\text {avg }}\right)$ in milk $(\mu \mathrm{g} / \mathrm{L})$ throughout the 24-hour sampling period, calculated as the AUC during the sampling period of 24 hours divided by 24 . The RID was calculated as the 
weight-adjusted AID ( $\mu \mathrm{g} / \mathrm{kg} /$ day) expressed as a percentage of the weight-adjusted maternal dose ( $\mu \mathrm{g} / \mathrm{kg} /$ day $)$.

For medications with short elimination half-lives, such as most triptans, it is often considered more relevant to report the infant dose ingested in a feed. ${ }^{11}$ Therefore, assuming an average infant milk consumption in a feed of $30 \mathrm{~mL} / \mathrm{kg},{ }^{10}$ we also estimated the absolute and relative maximum infant dose in a feed, calculated at $C_{\max }$ in milk, that is visualizing a worst-case scenario of exposure if the infant was breastfed at the time of the peak concentration in milk.

\section{3 | RESULTS}

A total of 19 breastfeeding women with migraine participated in the study. The mean age of the women was 32.8 years (range 26-41 years). Mean age of the infants was 9.4 months (range 6 weeks to 30 months), and their mean body-weight was $8.9 \mathrm{~kg}$ (range $4.8-15.5 \mathrm{~kg}$ ). Five of the women reported exclusive breastfeeding at the time of inclusion, whereas the remaining women were partially breastfeeding. The demographics of the mothers and their infants are summarized in Table 1.

All participants reported use of triptans for migraine treatment. Two women (individual 5 and 6) reported interchangeable use of two different triptans, whereas one woman (individual 12) used two different formulations of the same triptan (Table 2). These three women each volunteered to submit two sample sets, on two separate occasions at least one week apart. The other 16 women each submitted one sample set, resulting in a total of 22 breast milk sample sets; sumatriptan $(n=8)$, rizatriptan $(n=5)$, zolmitriptan $(n=4)$, eletriptan $(n=3)$, almotriptan $(n=1)$ and naratriptan $(n=1)$. The doses of the triptans administered were all within the recommended adult dose ranges for the respective drugs (Table 2).

Pharmacokinetic parameters in breast milk $\left(C_{\mathrm{avg}}, C_{\max }, t_{\max }\right.$, AUC and $t_{1 / 2}$ ) of the six triptans are presented in Table 2. Milk drug levels of the two active metabolites $\mathrm{N}$-desmethyleletriptan and N-desmethylzolmitriptan were very low to barely detectable

T A B L E 1 Demographics of the study participants $(n=19)$ and their infants $(n=19)$ at the time of study inclusion

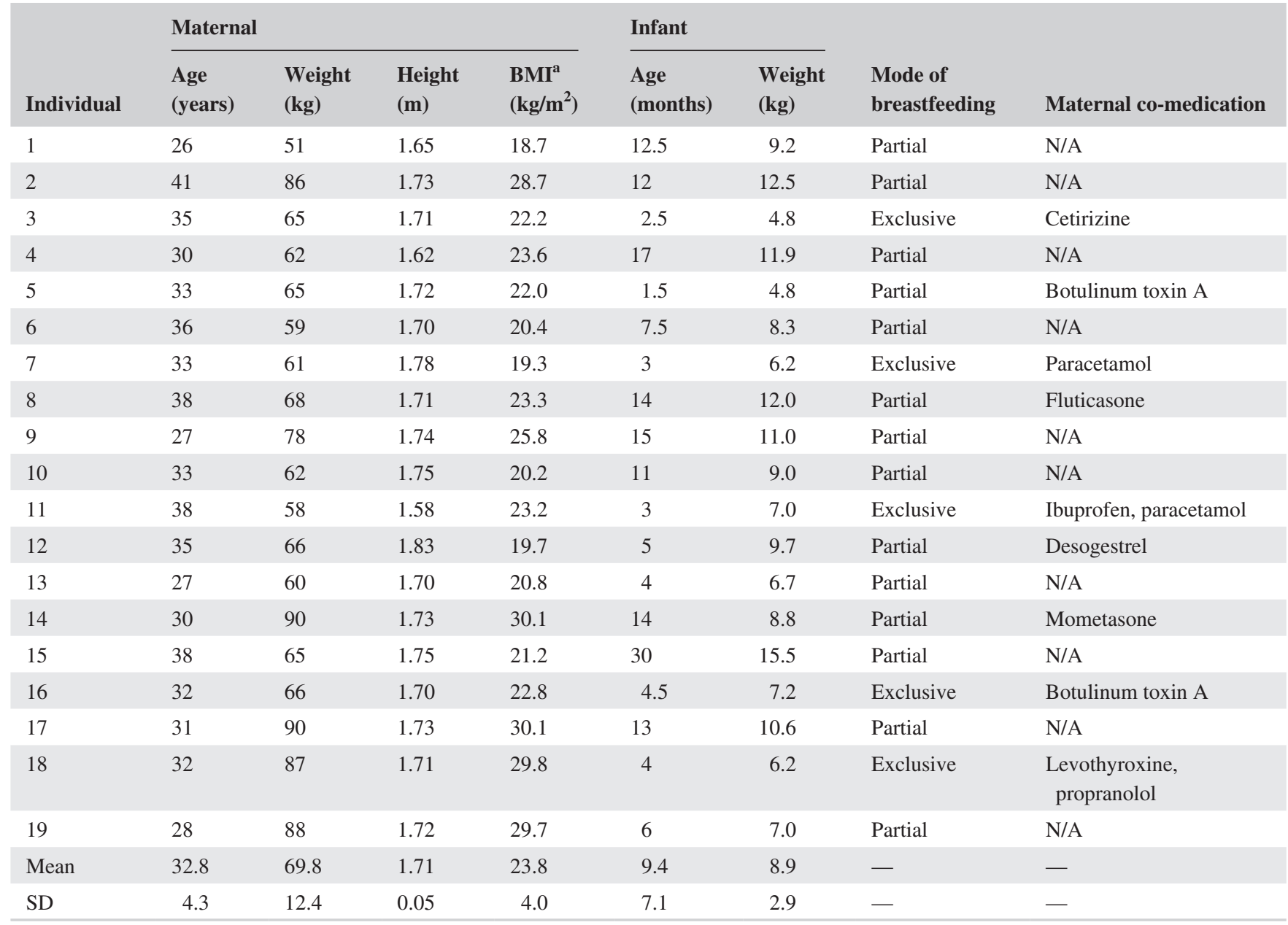

Note: Abbreviations: BMI, body mass index; N/A, not applicable; SD, standard deviation.

${ }^{\mathrm{a}} \mathrm{BMI}$ is the person's weight in kilograms divided by the square of the height in metres. 
T A B LE 2 Pharmacokinetic parameters of triptans in breast milk following intake of a single dose in 19 women $\left(n=22\right.$ sample sets $\left.{ }^{\mathrm{a}}\right)$, sorted according to triptan, administration form and dose

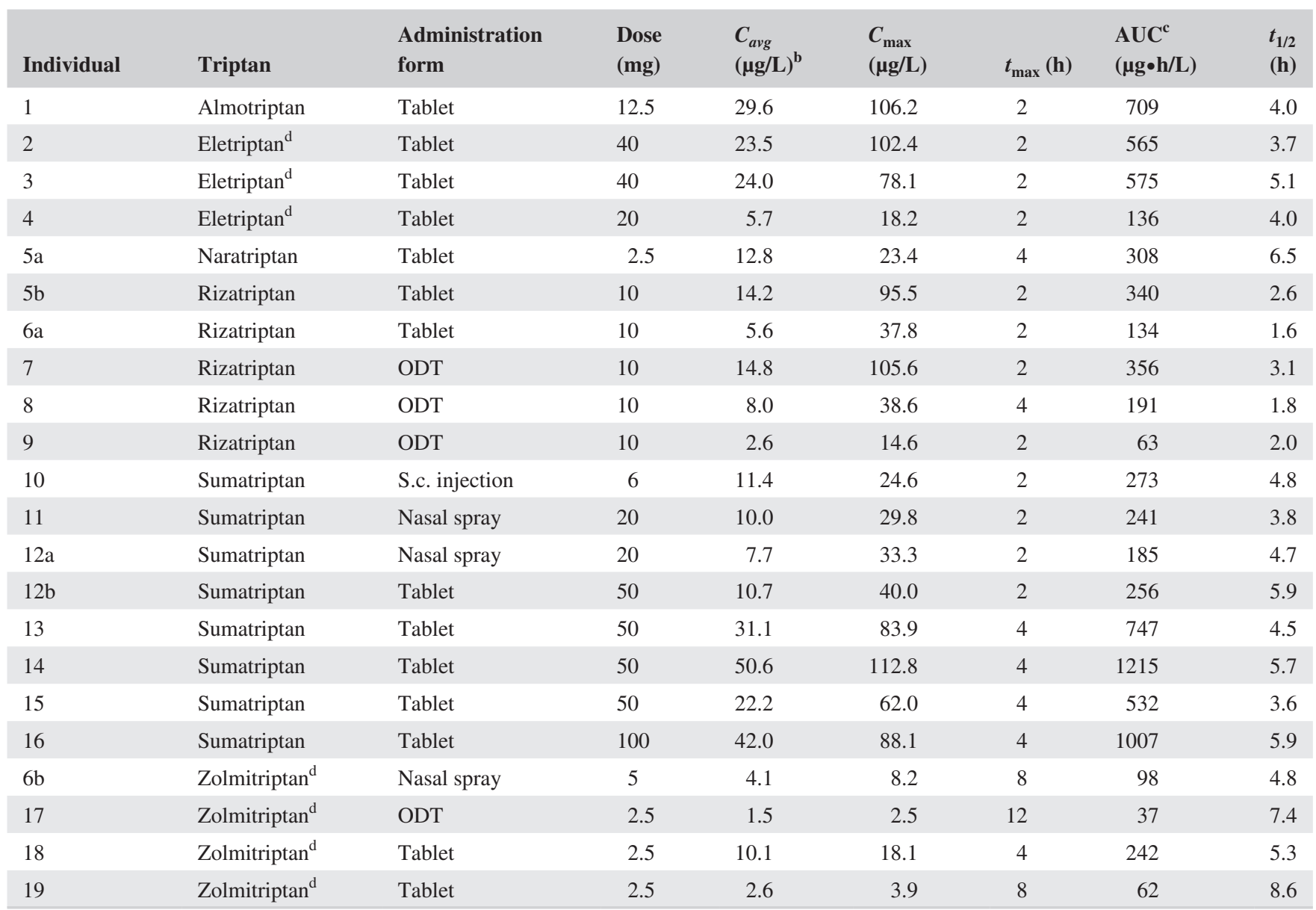

Note: Abbreviations: AUC, area under the milk concentration-time curve; $C_{\text {avg }}$, average concentration; $C_{\text {max }}$, maximum concentration; ODT, orally disintegrating tablet; s.c., subcutaneous; $t_{1 / 2}$, elimination half-life; $t_{\max }$, time to maximum concentration.

${ }^{a}$ Three of the study participants (individual 5, 6 and 12) each submitted two sample sets (a and b).

${ }^{\mathrm{b}}$ Average concentration in milk during the 24-hour sampling period (AUC/24).

${ }^{\mathrm{c}}$ The AUC values represent AUC from zero to infinity. The percentage extrapolated, representing the part of the area after the sample obtained 24 hours after dose, was below $8 \%$ with a few exceptions: For naratriptan (individual 5a), it was $10.0 \%$. For zolmitriptan, it was $21.9 \%$ in one individual (individual 18 ) and $16.6 \%$ in another individual (individual 17).

${ }^{\mathrm{d}}$ The active $\mathrm{N}$-desmethyl metabolite is not included in the calculations.

(below the assay LLOQ of $1 \mathrm{nM}$ ) and were not included in the calculations.

The individual triptan concentration-time profiles in breast milk during the 24-hour sampling period are depicted in Figure 1. In 19 out of the 22 samples, traces of triptans were still recovered in breast milk 24 hours after dose. For rizatriptan, however, there was no trace of drug in breast milk 24 hours after dose in three of five samples.

Table 3 shows the estimated theoretical AIDs and RIDs in a feed and in a day, respectively. Based on the $C_{\text {avg }}$ in milk throughout the 24-hour sampling period, estimated RID values (mean with range in parenthesis) were as follows: almotriptan $1.8 \%(-)$, eletriptan $0.6 \%(0.3 \%-0.8 \%)$, naratriptan $5.0 \%$ (-), rizatriptan $0.9 \%(0.3 \%-1.4 \%)$, sumatriptan $0.7 \%$ $(0.2 \%-1.8 \%)$ and zolmitriptan $2.1 \%(0.7 \%-5.3 \%)$.
When considering potential infant drug exposure based on $C_{\max }$ in milk during the day, calculated RID values in a day would still be $<10 \%$ of the maternal weight-adjusted dose for all the six triptans (mean with range in parenthesis): almotriptan $6.5 \%$ (-), eletriptan $2.0 \%$ (0.8\%-3.3\%), naratrip$\tan 9.1 \%(-)$, rizatriptan $5.6 \%(1.7 \%-9.7 \%)$, sumatriptan $1.8 \%$ (0.8\%-3.8\%) and zolmitriptan 3.6\% (1.4\%-9.4\%).

\section{4 | DISCUSSION}

The principal finding in the present study was that the excretion of triptans into breast milk seems to be low, with mean RID values varying from $0.6 \%$ to $0.7 \%$ for eletriptan and sumatriptan to $5.0 \%$ for naratriptan. Our results add to the sparse 

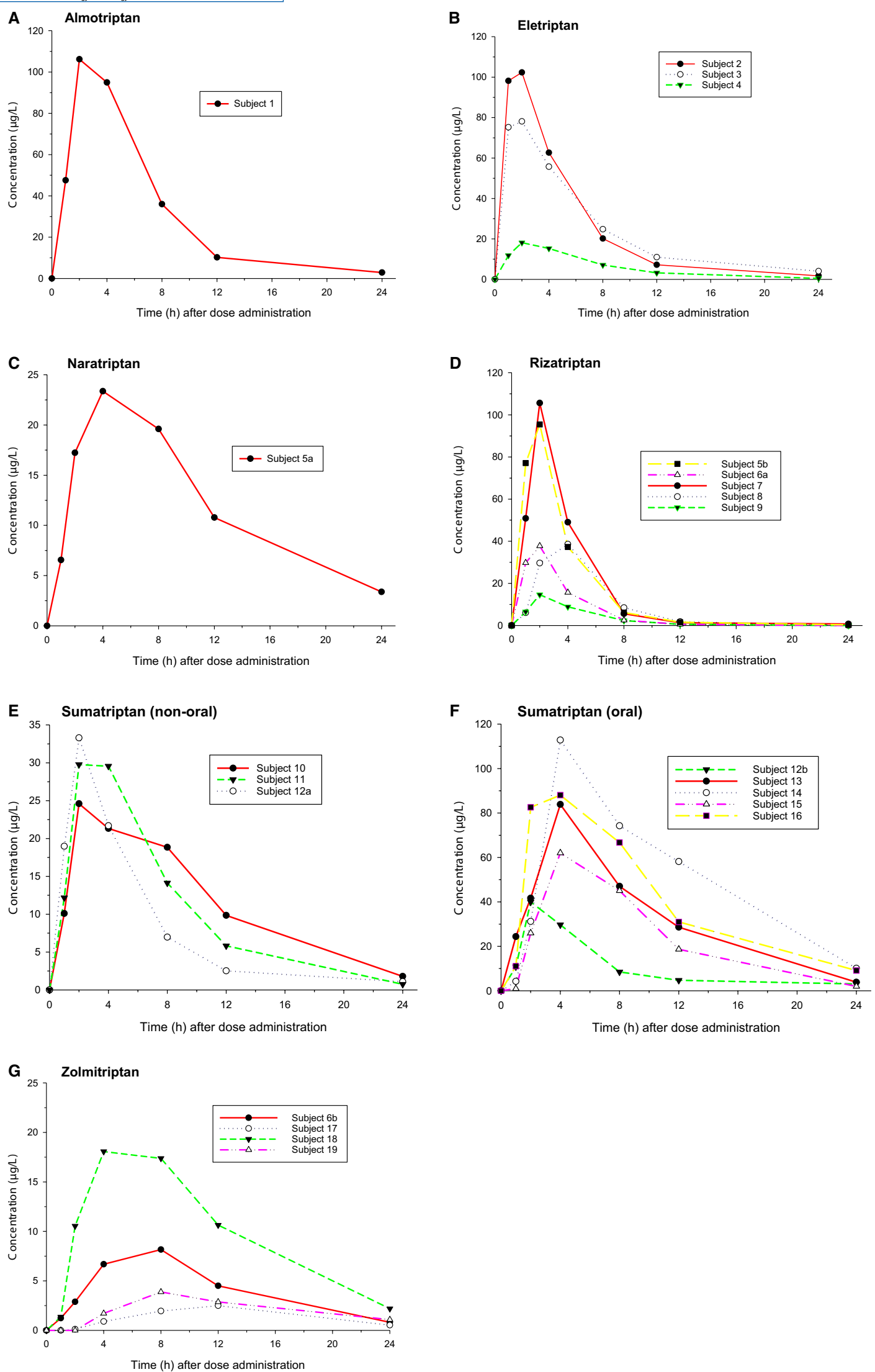

F I G U RE 1 Individual triptan concentration-time profiles in breast milk $(\mathrm{n}=22)$ following administration of a single dose in volunteers $(n=19)$ treated with almotriptan (A), eletriptan (B), naratriptan (C), rizatriptan (D), sumatriptan (E and F) and zolmitriptan (G). The milk concentrations are average values from the milk samples collected from both breasts 
existing knowledge on triptan excretion into breast milk and extend it to also include agents not previously studied. Moreover, this study is, to our knowledge, the first to analyse relevant active triptan metabolites, that is $\mathrm{N}$-desmethyleletriptan and $\mathrm{N}$-desmethylzolmitriptan, in breast milk.

This study has some limitations, but also some strengths, that should be addressed. One strength is that all participants submitted complete sample sets. This enabled us to monitor the triptan concentrations in breast milk at multiple time-points throughout the whole 24-hour sampling period. For each triptan, we covered all the different routes of administration available in Norway at the time of the study, with the exception of sumatriptan rectal suppositories (withdrawn from the market in 2017). Furthermore, we used a validated, sensitive and robust LC-MS/MS method for analysis.
Some limitations of this study also need to be discussed. The extent of transfer into milk of a given drug may vary according to the composition of the milk, with factors such as $\mathrm{pH}$ and lipid and protein content. ${ }^{10}$ Moreover, the lipid content varies within a feed, with hindmilk containing two- to threefold more fat than foremilk. ${ }^{12}$ Whereas the women in this study did not empty the breasts completely at each sampling, they collected mid-feed samples from each breast, as a means to obtain a representative sample of a feed. However, we did not analyse the milk samples with respect to $\mathrm{pH}$ and lipid and protein content and were thus unable to ascertain whether differences in milk composition affected the triptan concentrations.

Secondly, we did not collect concomitant blood samples from the participants, which prevented us from calculating $\mathrm{M} / \mathrm{P}$ ratios. Although the M/P ratio is useful for assessing

T A B LE 3 Estimated absolute and relative infant doses of triptans in 19 breastfed infants ( $\mathrm{n}=22$ sample sets), sorted according to triptan, maternal administration form and dose

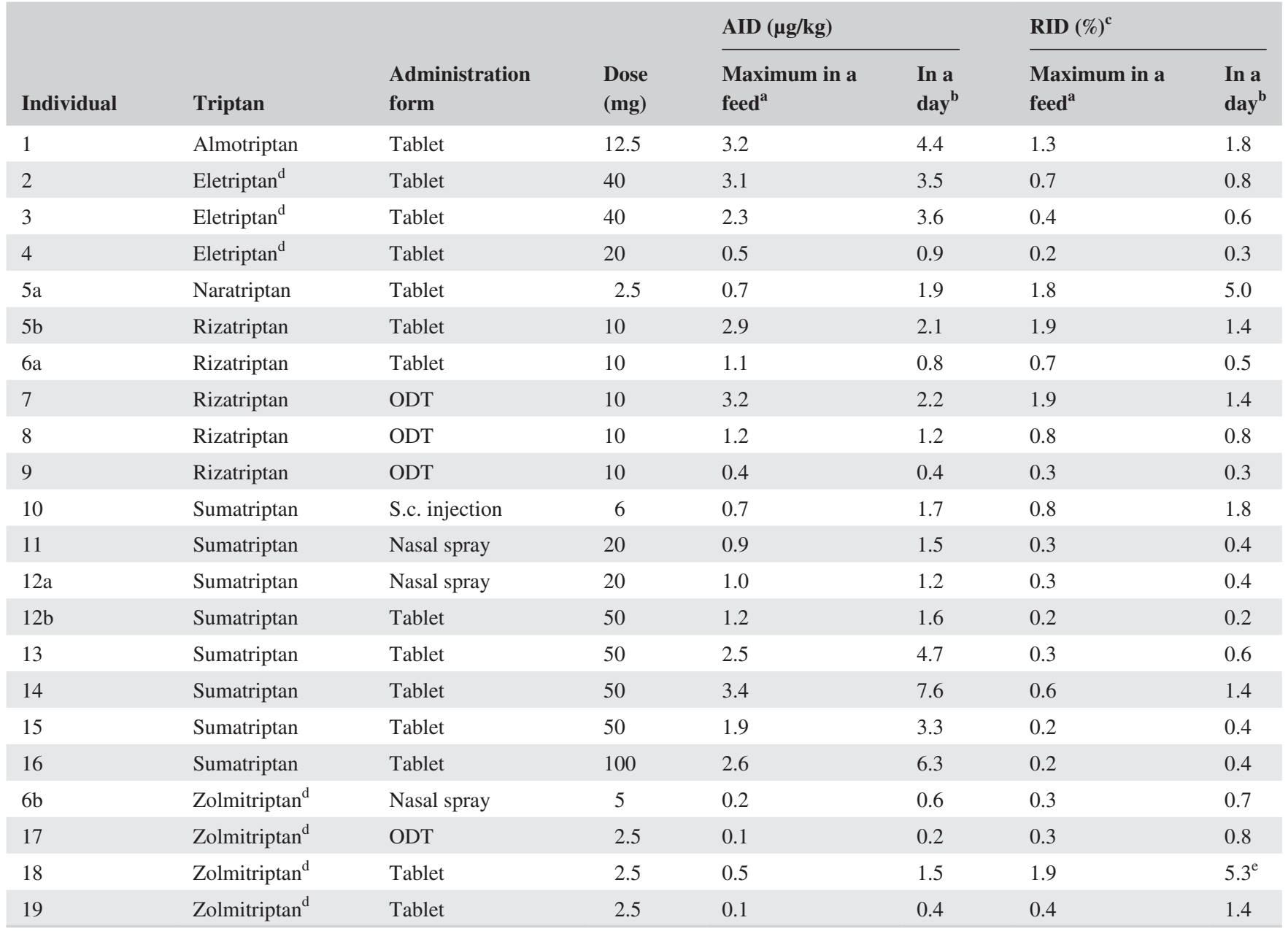

Note: Abbreviations: AID, absolute infant dose; ODT, orally disintegrating tablet; RID, relative infant dose; s.c., subcutaneous.

${ }^{a}$ Assuming an average infant milk intake of $30 \mathrm{~mL} / \mathrm{kg}$ body-weight peer feed, calculated at milk $C_{\max }$

${ }^{\mathrm{b}}$ Assuming exclusive breastfeeding with an average infant milk intake of $150 \mathrm{~mL} / \mathrm{kg}$ body-weight per day, calculated at milk $C_{\text {avg }}$.

${ }^{\mathrm{c}}$ Infant dose per kg body-weight expressed as a percentage of the maternal dose per kg body-weight.

${ }^{\mathrm{d}}$ The active $\mathrm{N}$-desmethyl metabolite is not included in the calculations.

${ }^{\mathrm{e}}$ Individual 18 reported concomitant use of propranolol. See text for further discussion. 
the extent to which a medication transfers from the mother's blood into her milk, it is not a clinically applicable variable to predict the risk of infant drug exposure. As the amount of drug transfer to milk is largely determined by the drug level in maternal plasma, even medications with a high M/P ratio may still result in low total drug milk concentrations if the maternal plasma concentration is low. Collection of concomitant blood samples could also have confirmed participants' adherence to dose intake. Nevertheless, we had no reason to suspect non-adherence in this study as the displayed concentration-time curves in milk for all volunteers were in concordance with intake of a single dose, with no drug present at baseline ( 0 hours). The FDA recommendations underline the importance of minimizing the burden of data collection while at the same time obtaining adequate data, and generally recommend milk-only studies unless there is a specified reason to conduct another type of clinical lactation study. ${ }^{9}$

Thirdly, we had to rely on participants' self-reporting of timing, as we were not able to verify that the women conducted the breast milk sampling at the assigned times. Neither could we confirm that they followed the instructions of freezing the samples immediately after sampling. In both situations, deviations from the instructions could potentially influence the estimates presented. Finally, the relatively small study size means that generalization of the results must be made with caution, especially for the substances used by only one, or a few of the participants. Our results must be interpreted with these limitations in mind.

Our findings show that all the six triptans under study were found in breast milk after administration of a single dose. As illustrated in Figure 1, there was a relatively large interindividual variability in the concentrations of each specific triptan, even when taking into consideration dose and administration form. Of special note, we observed a $28 \%$ higher $C_{\max }$ in individual 14 taking an oral dose of $50 \mathrm{mg}$ sumatriptan than in individual 16 taking the double dose. In individual 18 taking zolmitriptan $2.5 \mathrm{mg}$ we observed substantially higher $C_{\max }$ and AUC values in milk compared with the other participants using zolmitriptan in equal or higher doses (see Table 2). This woman reported concomitant use of propranolol for migraine prevention in a total daily dose of $80 \mathrm{mg}$. In a randomized crossover study including 12 healthy volunteers, pretreatment with propranolol $160 \mathrm{mg} /$ day for 7 days was found to increase mean zolmitriptan $C_{\max }$ and AUC by $56 \%$ and $37 \%$, respectively. ${ }^{13}$ Thus, concomitant use of propranolol might explain the observed higher $C_{\max }$ and AUC in milk in individual 18. These findings demonstrate that the individual disposition of a drug is highly variable. As most lactation studies are conducted in a relatively small number of women, individual variances in maternal pharmacokinetics must always be considered when extrapolating individuallevel end-points to a population level.

Only one of the participants in our study reported use of sumatriptan by subcutaneous injection, which also was the route of administration examined in the previous study of sumatriptan excretion in milk. ${ }^{6}$ The observed $t_{\max }$ ( 2 hours) in our individual was in agreement with the values reported previously (median 2.5 hours; range 1.7-3.5 hours). In the current study, however, we observed a substantially lower $C_{\max }(24.6 \mu \mathrm{g} / \mathrm{L})$ compared with the previous study where the mean $C_{\max }$ was $87.2 \mu \mathrm{g} / \mathrm{L}$ (range 61.9-112.5 $\mu \mathrm{g} / \mathrm{L}$ ). Moreover, the $\mathrm{t}_{1 / 2}$ in our study individual (4.8 hours) was prolonged compared with the previously reported mean $t_{1 / 2}$ of 2.2 hours (range 1.2-3.1 hours). Their estimated RID (mean $3.5 \%$; 95\% CI $0.3 \%-6.7 \%$ ) was based on the cumulative excretion of drug into milk, ${ }^{6}$ making direct comparison with our results difficult. In contrast, the estimated RID in our study was $0.7 \%$ based on the average concentration throughout the day.

The presence of active metabolites should always be taken into account when assessing possible risks to an infant following drug exposure through breastfeeding. The metabolite concentrations should also be included in the estimations of the infant dose. In our material, the milk concentrations of the active metabolites of eletriptan and zolmitriptan were very low to barely detectable (below the assay LLOQ). As such low concentrations would not be expected to contribute significantly to the overall infant drug exposure, they were not included in the calculations.

An RID of 5\%-10\% may be considered a threshold value in safety assessment. ${ }^{10,14-16}$ For most medications (with the exception of highly toxic agents), an RID below $10 \%$ would generally be considered compatible with breastfeeding. ${ }^{7}$ For all the six triptans in this study, the estimated RID values were well below this level, indicating that infant drug exposure of triptans through breastfeeding is low. Moreover, if the oral bioavailability in infants is the same as in adults, systemic exposure would be further reduced. In adults, triptans have an oral bioavailability ranging from $14 \%$ (sumatriptan) to $74 \%$ (naratriptan) and have relatively short half-lives, ranging from 2 hours (sumatriptan and rizatriptan) to 6 hours (naratriptan). Frovatriptan differs from the other agents in this class with a significantly prolonged elimination half-life of 26 hours. $^{2}$

Even when $C_{\max }$ in milk was used to calculate RID values, that is depicting a worst-case scenario for potential infant exposure, they were still below the threshold of $10 \%$ of the maternal dose. For rizatriptan, with its demonstrated short $t_{1 / 2}$, the estimated RID in a feed at $C_{\max }$ equalled or exceeded the corresponding RID in a day calculated at $C_{\text {avg }}$. Thus, withholding breastfeeding for 4 hours after intake of rizatriptan would cause a larger decrease in exposure than for the other triptans. 
Disregarding the individual zolmitriptan RID of 5.3\% in individual 18, naratriptan was the substance with the highest estimated RID (5.0\%) in this study. Although the estimated RID is based on only one individual, considering naratriptan's high oral bioavailability (74\%) and thus its higher potential of infant exposure, caution might be advisable in mothers breastfeeding newborn infants. Some caution with generalization is also advisable for almotriptan, as only one individual used this triptan, although the calculated RID was reassuringly low.

Sources differ in their recommendations as to whether it is necessary to withhold breastfeeding after maternal treatment with triptans. The recommendations in the product monographs for these agents are in general precautious due to lack of breastfeeding data and recommend for most triptans that breastfeeding should be avoided for 24 hours after treatment (see Table S1). A less strict warning is given in the product monograph for sumatriptan, stating that infant drug exposure can be minimized by avoiding breastfeeding for 12 hours after treatment. In the aforementioned study on sumatriptan, the authors suggested expressing and discarding milk for 8 hours after the dose as a means to reduce the amount of drug ingested by the breastfed infant. ${ }^{6}$ The findings from the present study are generally reassuring, and we consider it being a too conservative approach to categorically advise women to withhold breastfeeding. Instead, we suggest that the mother may resume breastfeeding as soon as practically feasible after she recovers from the migraine attack, without discarding milk. As a general rule of thumb, if equally effective for the mother, triptans with shorter elimination half-lives should be preferred during breastfeeding, as they are eliminated more rapidly from maternal plasma, and consequently also from the milk compartment.

In conclusion, our findings demonstrate low excretion into breast milk of the triptans studied, including relevant active metabolites. The estimated RID values indicate that infant drug exposure is low and suggest that use of the triptans included in the present study is compatible with breastfeeding. Still, for each patient, an individual assessment needs to be made. Caution might be warranted in women breastfeeding newborn infants when using naratriptan.

\section{ACKNOWLEDGEMENTS}

The study was funded by a research grant from the Northern Norway Regional Health Authority (Helse Nord RHF) (Project ID: SFP1248-15). We thank all the women who participated in the study. We acknowledge the assistance of RELIS (www.tryggmammamedisin.no) in the recruitment process, and Siri von Krogh and Anne Christine Poole who promoted the study. We also thank the Mothers' Milk Bank, University Hospital of North Norway, Troms $\varnothing$, Norway for donation of human donor milk. The publication charges for this article have been funded by a grant from UiT - The Arctic University of Norway, Troms $\varnothing$, Norway.

\section{CONFLICT OF INTEREST}

The authors declare no conflicts of interest.

\section{ORCID}

Siri Amundsen (D) https://orcid.org/0000-0002-2786-9852

Hedvig Nordeng (D) https://orcid.org/0000-0001-6361-2918

Ole-Martin Fuskevåg (iD https://orcid.

org/0000-0003-4733-0499

Georg Sager (DD https://orcid.org/0000-0003-1675-5881

Olav Spigset (iD https://orcid.org/0000-0001-7902-9014

\section{REFERENCES}

1. Cameron C, Kelly S, Hsieh S-C, et al. Triptans in the acute treatment of migraine: a systematic review and network meta-analysis. Headache 2015;55(Suppl 4):221-235.

2. Johnston MM, Rapoport AM. Triptans for the management of migraine. Drugs 2010;70:1505-1518.

3. Hoshiyama E, Tatsumoto M, Iwanami $\mathrm{H}$, et al. Postpartum migraines: a long-term prospective study. Intern Med. 2012;51:3119-3123.

4. Sances G, Granella F, Nappi RE, et al. Course of migraine during pregnancy and postpartum: a prospective study. Cephalalgia 2003;23:197-205.

5. Kvisvik EV, Stovner LJ, Helde G, Bovim G, Linde M. Headache and migraine during pregnancy and puerperium: the MIGRAstudy. J Headache Pain. 2011;12:443-451.

6. Wojnar-Horton RE, Hackett LP, Yapp P, Dusci LJ, Paech M, Ilett KF. Distribution and excretion of sumatriptan in human milk. $\mathrm{Br} \mathrm{J}$ Clin Pharmacol. 1996;41:217-221.

7. Hale TW. Hale's Medications \& Mothers' Milk: A Manual of Lactational Pharmacology, 19th edn. New York, NY: Springer Publishing Company; 2021.

8. Tveden-Nyborg $\mathrm{P}$, Bergmann TK, Jessen N, Simonsen U, Lykkesfeldt J. BCPT policy for experimental and clinical studies. Basic Clin Pharmacol Toxicol. 2021;128:4-8.

9. U.S. Food and Drug Administration (FDA). Clinical lactation studies: considerations for study design, 2019. https://www.fda.gov/ media/124749/download. Accessed August 30, 2020

10. Bennett PN. Drugs and Human Lactation, 2nd edn. Amsterdam: Elsevier; 1996

11. Spigset $\mathrm{O}$, Hägg $\mathrm{S}$. Analgesics and breast-feeding: safety considerations. Paediatr Drugs. 2000;2:223-238.

12. Saarela T, Kokkonen J, Koivisto M. Macronutrient and energy contents of human milk fractions during the first six months of lactation. Acta Paediatr. 2005;94:1176-1181.

13. Peck RW, Seaber EJ, Dixon R, et al. The interaction between propranolol and the novel antimigraine agent zolmitriptan (311C90). Br J Clin Pharmacol. 1997;44:595-599.

14. Larsen ER, Damkier P, Pedersen LH, et al. Use of psychotropic drugs during pregnancy and breast-feeding. Acta Psychiatr Scand 2015; 132:1-28

15. Anderson PO, Sauberan JB. Modeling drug passage into human milk. Clin Pharmacol Ther. 2016;100:42-52. 
16. Verstegen RHJ, Anderson PO, Ito S. Infant drug exposure via breast milk. Br J Clin Pharmacol. 2020. https://doi.org/10.1111/ bcp.14538. Online ahead of print.

\section{SUPPORTING INFORMATION}

Additional supporting information may be found online in the Supporting Information section.
How to cite this article: Amundsen S, Nordeng H, Fuskevåg O-M, Nordmo E, Sager G, Spigset O. Transfer of triptans into human breast milk and estimation of infant drug exposure through breastfeeding. Basic Clin Pharmacol Toxicol. 2021;128:795-804. https://doi.org/10.1111/bcpt.13579 